\title{
Permanent Magnet Synchronous Machine Drive Control Using Analog Hall-Effect Sensors
}

\author{
David Reigosa ${ }^{*}$, Daniel Fernandez ${ }^{*}$, Cristina Gonzalez ${ }^{*}$, Sang Bin Lee ${ }^{* *}$ and Fernando Briz ${ }^{*}$ \\ "University of Oviedo. Dept. of Elect., Computer \& System Engineering, Gijón, 33204, Spain. \\ Korea University, Dept. of Elect. Engineering, Seoul, Korea. \\ diazdavid@uniovi.es, fernandezalodaniel@uniovi.es, gmoralcristina@uniovi.es, sangbinlee@korea.ac.kr, \\ fernando@isa.uniovi.es
}

\begin{abstract}
Control of permanent magnet synchronous machines (PMSMs) requires rotor position measurement/estimation, as well as the magnet polarity detection for startup of the machine. The rotor position of PMSMs is typically measured using a speed/position sensor (e.g. an encoder), while the magnet polarity is commonly measured using digital hall-effect sensors. Since simplifying the system can help improve reliability and reduce the cost associated with the measurements, the use of low cost analog hall-effect sensors for estimation of the PM position and polarity for control of PMSMs drives is evaluated in this paper. Experimental results performed on a $7.5 \mathrm{~kW}$ motor confirm that analog hall-effect sensors can replace both speed/position sensors and digital hall-effect sensors, while maintaining adequate performance of the drive at lower cost. ${ }^{1}$
\end{abstract}

Index Terms - Permanent magnet synchronous machines, rotor position estimation, Hall-effect sensors.

\section{Introduction}

Permanent magnet synchronous machines (PMSMs) have become very popular during the last decades due to their high efficiency, high power density and superior dynamic response compared with other types of machines, e.g. induction or synchronous reluctance machines. Control of PMSMs requires rotor position measurement/estimation, as well as knowledge of the magnet polarity prior to startup of the machine [1]-[5]. The rotor position of PMSMs is typically measured using encoders [6]-[9], while the magnet polarity is commonly measured using digital hall-effect sensors [10][15]. Consequently, commercial PMSMs are often equipped with both types of sensors [6]-[9].

Cost of encoders and the associated cabling and interfaces can account for a significant portion of the overall drive cost, especially in low-power applications. In addition, the sensor (e.g. moving parts, coupling...) and the associated cabling and connectors can be a source of failure, reducing the drive reliability. For these reasons, the elimination of the position/speed sensor is always desirable.

Hall-effect sensors are commonly used in permanent magnet machines, e.g. brushless DC (BLDC), interior PMSMs

\footnotetext{
${ }^{1}$ This work was supported in part by Spanish Ministry of Education, Culture and Sports through "José Castillejo Program" under grant PX15/00354, by Regional Ministry of Education, Culture and Sport of the Principality of Asturias through "Severo Ochoa Program" under Grant BP-13067 and by the Research, Technological Development and Innovation Programs of the Spanish Ministry Economy and Competitiveness, under grant MINECO-17ENE2016-80047-R.
}

(IPMSMs), surface PMSMs (SPMSMs)..., both for magnet polarity and position estimation [10]-[15]. Digital hall-effect sensors provide position estimations with a resolution of 60 electrical degrees. This resolution is adequate for BLDC drives fed with 120 electrical degree square-waveform voltages [10]-[15], but is insufficient for torque control of $\mathrm{AC}$ drives using IPMSMs and SPMSMs, and also for motion control. Methods to improve the resolution of hall-effect sensors have been proposed in [11], [16] and [17]. However, these methods present limitations during machine startup, fast acceleration/deceleration processes, or at very low speeds. Strategies to minimize these limitations are analyzed in [18].

An alternative to the use of speed/position and digital halleffect sensors are sensorless techniques [24]-[33]. Sensorless methods can be roughly classified into fundamental excitation based techniques (back-EMF) [24]-[29] and saliency tracking based techniques [30]-[33]. Fundamental excitation based techniques are used in the mid-high speed region. Unfortunately their performance degrades as the speed decreases since the back-EMF is proportional to the speed. Furthermore, they cannot operate at very low or zero speed, and in position control. To overcome the limitations of fundamental excitation based techniques, saliency tracking based techniques have been proposed. Saliency tracking based techniques allow position/speed estimation in the entire speed range of the machine even at very low and zero speeds. Unfortunately, saturation-induced saliencies, secondary saliencies and cross-coupling reduce the accuracy of these methods to unacceptable levels and even compromise the stable operation of the drive [31]-[33].

The use of low cost analog hall-effect sensors for torque and motion control of PMSM drives [6]-[9], [19]-[23] and [42] is investigated in this paper. Contrary to previous proposals [19]-[23], the proposed method uses the magnetic flux density complex vector. This enables the use of powerful and efficient synchronous reference frame phase-lock loop (SRP-PLL) based methods [40]-[41]. In the methods proposed in [19]-[23], the sensors were arbitrarily aligned in a radial direction. However, no analysis was provided supporting this selection. 3D sensors will be used in this work to evaluate the sensitivity of the method to the sensors' orientation. Implementation issues which affect to the accuracy of the estimated speed/position, including offsets in the sensors, variations in the sensors' gains, temperature dependence of the sensors, assembling tolerances, effects of the stator currents, etc., will also be analyzed. 


\begin{tabular}{|l|l|}
\hline \multicolumn{2}{|c|}{ Table I. Test machine parameters (IPMSM) } \\
\hline$P_{\text {RATED }}(\mathrm{kW})$ & 7.5 \\
\hline$I_{\text {RATED }}(\mathrm{A})$ & 14 \\
\hline$\omega_{\text {RATED }}(\mathrm{rpm})$ & 1000 \\
\hline Stator slots & 36, unskewed \\
\hline Poles & 6 \\
\hline Rotor radius $(\mathrm{mm})$ & 54.2 \\
\hline Magnets & $\mathrm{N} 42 \mathrm{SH}$ \\
\hline Magnet dimensions: width, height and length $(\mathrm{mm})$ & $42 \times 6 \times 10$ \\
\hline Magnet position from shaft center $(\mathrm{mm})$ & 44.6 \\
\hline Magnet position, $x y z(\mathrm{~mm})$ & $0,0,6$ \\
\hline Airgap length $(\mathrm{mm})$ & 0.8 \\
\hline Inner stator radius $(\mathrm{mm})$ & 55 \\
\hline Outer stator radius $(\mathrm{mm})$ & 88 \\
\hline
\end{tabular}

\begin{tabular}{|l|l|}
\hline \multicolumn{2}{|c|}{ Table II. Load machine parameters } \\
\hline$P_{\text {RATED }}(\mathrm{kW})$ & 45 \\
\hline$I_{\text {RATED }}(\mathrm{A})$ & 78.81 \\
\hline$\omega_{\text {RATED }}(\mathrm{rpm})$ & 2970 \\
\hline
\end{tabular}

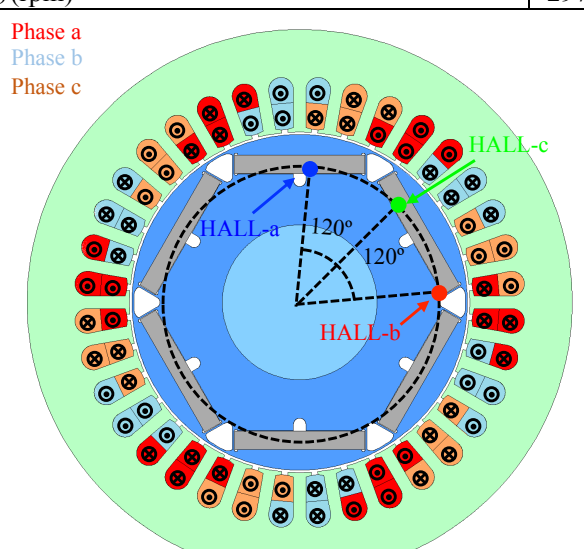

a)

b)

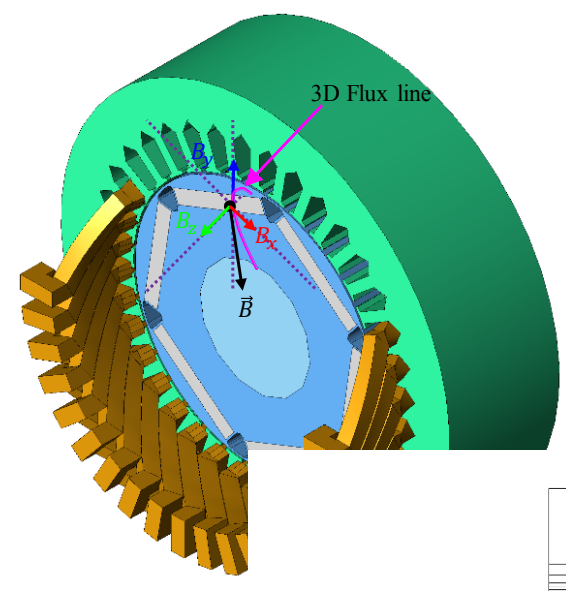

c)

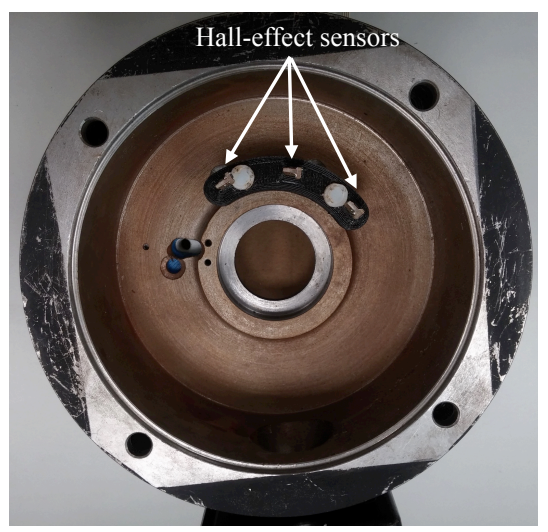

d)
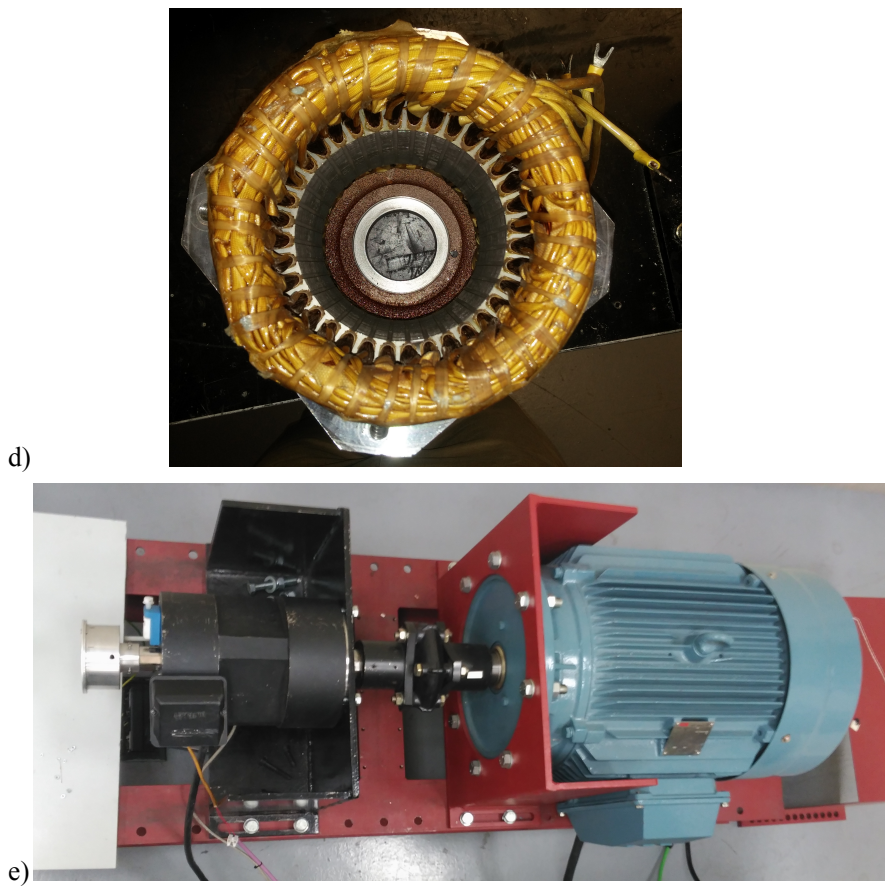

Fig. 1.- Machine and experimental setup. a) 2D and b) 3D schematic representation of the machine and sensors location. $x, y$ and $z$ correspond to the tangential, radial and axial directions respectively. c) Machine end shield with the hall-effect sensors, d) stator and e) test bench.

The paper is organized as follows: principles of the rotor position estimation using analog hall-effect sensors and experimental results to demonstrate the viability of the concepts are presented in section II. A discussion of practical implementation issues is presented in section III, while conclusions are provided in section IV.

\section{Speed/position estimation using analog hall-effect sensors}

This section analyzes the principles of speed/position estimation using analog hall-effect sensors. Details of the test machine are shown in Table I. Fig. 1a shows a schematic representation of the PMSM that will be used. Hall-sensors are aligned with the field induced by the current flowing throughout phases $a, b$ and $c$ respectively. The phase shift among sensors is therefore 120 electrical degrees, which corresponds to 40 mechanical degrees in a 6-pole machine. Standard hall-effect sensors used in PMSM drives measure the magnetic flux density along one direction, i.e. they are 1D sensors. 3D sensors have been used in this work however, to determine the optimal sensor angle. These sensors provide measurements in the $x, y$ and $z$ directions shown in Fig. 1b, direction $y$ corresponding to the normal alignment of $1 \mathrm{D}$ sensors [6]-[9]. Hall-effect sensors are attached to the end shield as shown in Fig. 1c. Stator of the machine is shown in Fig. 1d. Fig. 1e shows the test bench consisting of the test machine and a load machine. Details of the load machine are shown in Table II [49].

Fig. 2 shows the signal processing used for speed and position estimation. The signal processing can be applied to either the sensors in the $x, y$ or $z$ directions. However, from the analysis performed in this work it was determined that the 


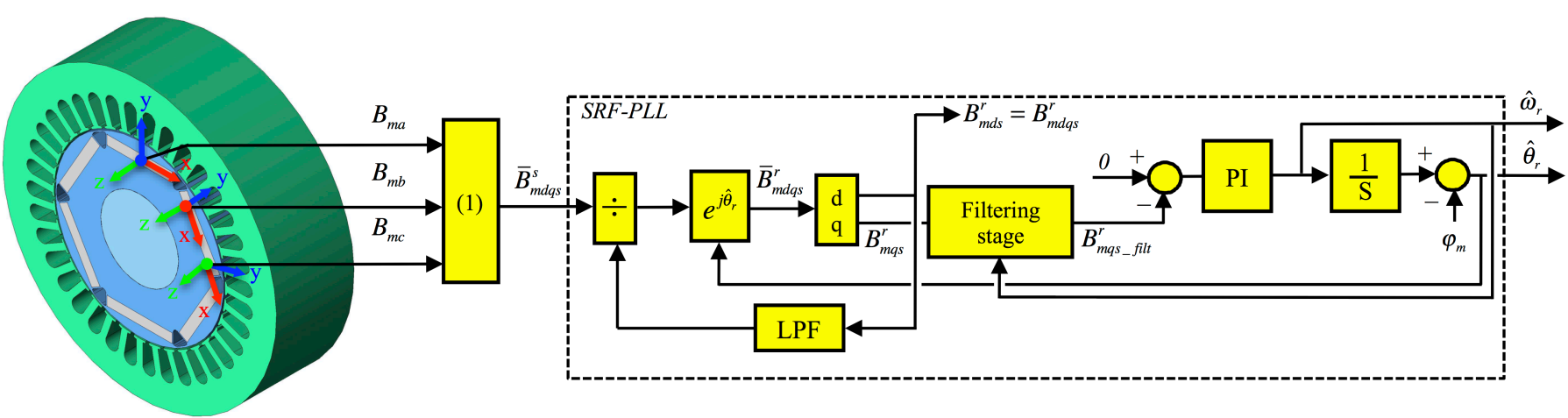

Fig. 2.- Coordinate system for each sensor ( $x=$ tangential direction, $y=$ radial direction, $z=$ axial direction) and signal processing used for speed and position estimation. Input to the SFR-PLL is normalized to have a unit magnitude. By doing this, SFR-PLL dynamics become independent from the input signal magnitude.

magnetic flux density in the $z$-axis direction is significantly smaller than in the $x$ and $y$-axis directions. This places serious concerns on the reliability of position/speed estimation using $z$-axis measurements, this option not being therefore discussed further. Connection between the hall-effect sensors and the drive is made using a standard shielded cable [44].

$$
\begin{aligned}
\bar{B}_{m d q s}^{s} & =2 / 3\left(B_{m a}+a B_{m b}+a^{2} B_{m c}\right)= \\
& =B_{m d q s}^{s} e^{j\left(\theta_{r}+\varphi_{m}\right)}=B_{m d q s}^{s} e^{j\left(\omega_{r} r+\varphi_{m}\right)} \\
\bar{B}_{m d q s}^{r} & =\bar{B}_{m d q s}^{s} e^{-j \theta_{r}}
\end{aligned}
$$

A magnetic flux density complex vector, (1) $\bar{B}_{m d q s}^{s}$, can be defined using the measurements of the magnetic flux densities from all the three hall-effects sensors. This transformation can be applied to the measurements in $x$-axis or $y$-axis direction. $B_{m a}, B_{m b}$ and $B_{m c}$, are the magnetic flux densities measured by sensors $a, b$ and $c$ along the $m$ direction, where $m$ represents $x$ or $y$-axis direction. $B_{m d q s}^{s}$ is the magnitude of $\bar{B}_{m d q s}^{s}, \theta_{r}$ is the angle of the rotor and $\varphi_{m}$ is the angle between the field produced by the stator current (which has a radial direction) and the measuring direction of field sensors in the $x$ and $y$ axis, i.e. $\varphi_{x}=90$ and $\varphi_{y}=0$.

It can be observed from (1) that $\bar{B}_{m d q s}^{r}$ is a complex vector which contains the information on rotor speed and position. The block diagram of the proposed rotor speed and position estimation method is shown in Fig. 4. The inputs to the control block diagram are the hall sensors measurements, the resulting complex vector $\bar{B}_{m d q s}^{s},(1)$, feeds an SRP-PLL which includes a filter "in the loop" stage [40]. The magnetic flux density complex vector in the stator reference frame $\left(\bar{B}_{m \text { dqs }}^{s}\right)$ is normalized and transformed to a reference frame synchronous with the rotor $\left(\bar{B}_{m d q s}^{r}\right)$ using the estimated rotor position, where the transformation from the stator $\left(d q^{s}\right)$ to the rotor reference frame $\left(d q^{r}\right)$ is defined by (2). The resulting $q$-axis component of $\bar{B}_{m d q s}^{r}\left(B_{m q s}^{r}\right)$ is processed before feeding the PLL PI controller. Signal processing is discussed in detail in section III. A PI controller is used to drive the $q$-axis component of $\bar{B}_{m d q s}^{r}$ to zero, and the estimated magnetic flux density complex vector is aligned to the rotor $d$-axis. Discussion of the PLL tuning can be found in Appendix I. The output of the PI controller is the estimated electrical speed, the electrical rotor position being obtained by integration. As already discussed, decoupling of the $x$ or $y$ -

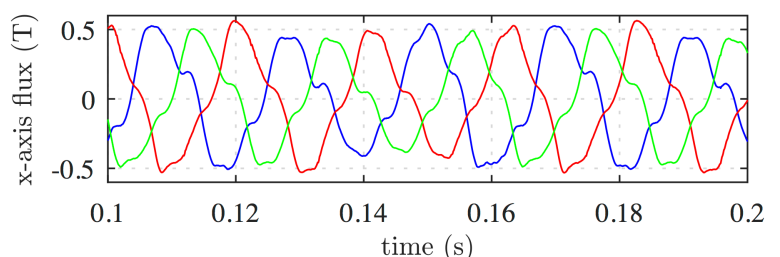

a)

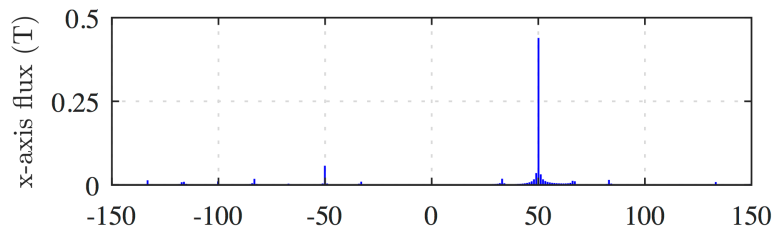

b)

$(\mathrm{Hz})$

Fig. 3.- a) $x$-axis flux measurements by the three hall effect sensors; b) FFT of the resulting magnetic flux complex vector, $\bar{B}_{x d q s}^{s}(1)$. Sensor position: $\mathrm{x}=0 \mathrm{~mm}, \mathrm{y}=0 \mathrm{~mm}, \mathrm{z}=5 \mathrm{~mm}$. $\omega_{r}=1 \mathrm{pu}$ and $i_{d q}=0 \mathrm{pu}$.
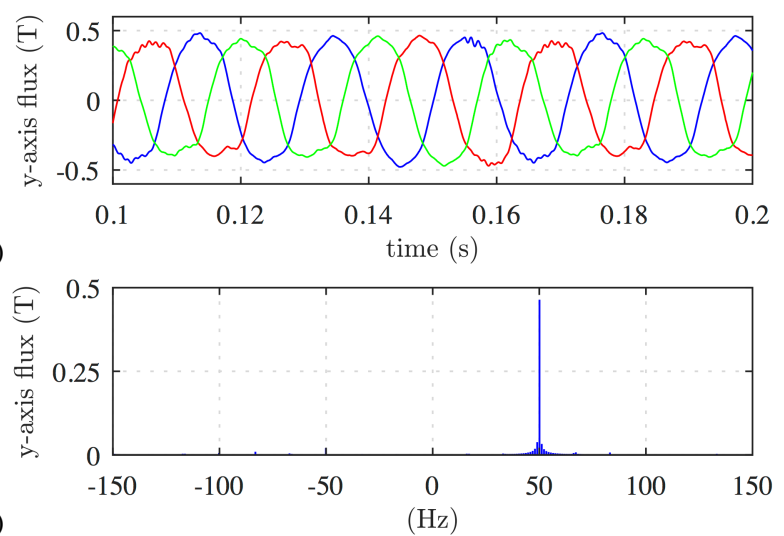

Fig. 4.- Same results as in Fig. 5 but for the $y$-axis direction.

axis angular offsets ( $\varphi_{y}=0$ and $\varphi_{x}=90$ deg.) is required. It is noted that only the angle of $\bar{B}_{m d q s}^{r}$ is relevant for position estimation purposes, the magnitude of $\bar{B}_{\text {mdqs }}^{r}$ will influence the dynamic response of the SRF_PLL, and therefore, must be considered for the gain selection.

In the experiments following, the machine was rotated by a prime mover and fed from a current regulated power converter using the estimated position for coordinate rotations and with $i_{d q}=0$ pu. Fig. 3 and Fig. 4 show the measured magnetic flux densities and the FFT of $\bar{B}_{m d q s}^{r}$ along $x$-axis and $y$-axis directions, respectively. It is observed from Fig. 3 and Fig. 4 that the magnitude of $\bar{B}_{m d q s}^{r}$ is similar in both axes, but slightly higher in the $y$-axis. However, the harmonic content (noise) of 
$\bar{B}_{x d q s}^{r}$ is larger than that of $\bar{B}_{y d q s}^{r}$. Consequently, $y$-axis offers a better signal-to-noise ratio, and therefore, is expected to provide more accurate estimates.
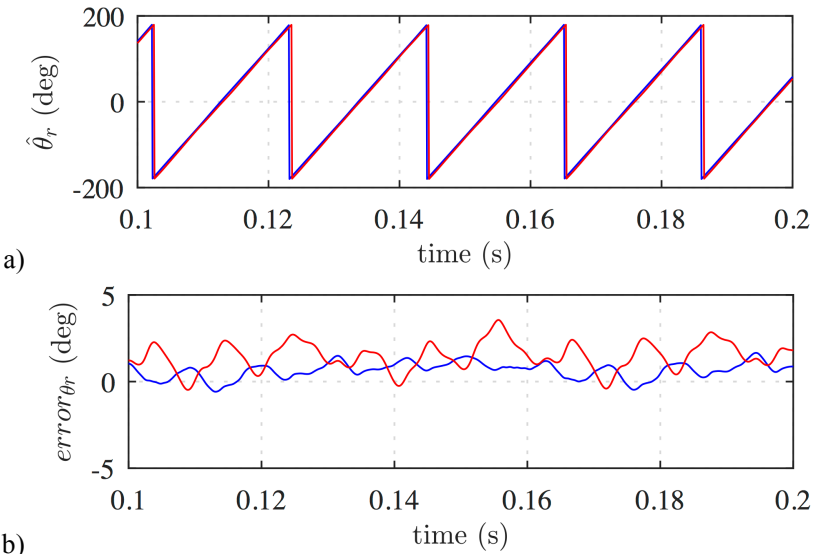

Fig. 5.- a) Estimated position, and, b) position error, when using $\bar{B}_{x d q s}^{s}$ (red) and $\bar{B}_{y d q s}^{s}$ (blue). Sensor position: $\mathrm{x}=0 \mathrm{~mm}, \mathrm{y}=0 \mathrm{~mm}, \mathrm{z}=5 \mathrm{~mm} . \omega_{r}=1 \mathrm{pu}$ and $i_{d q}=0 \mathrm{pu}$.
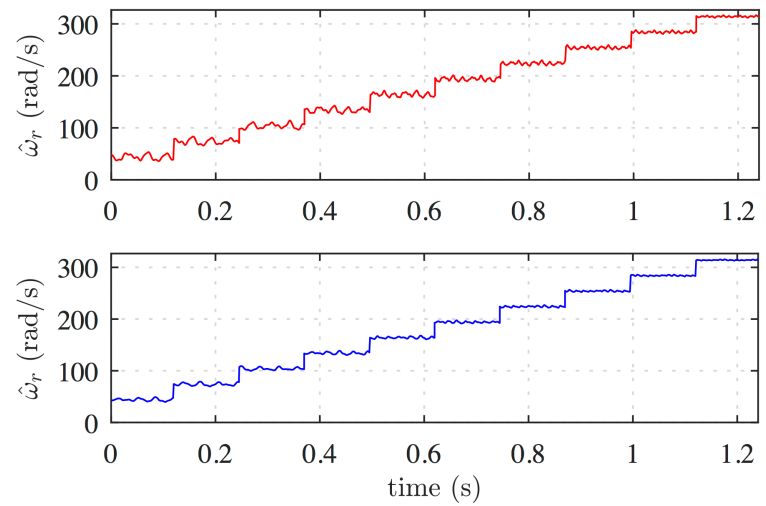

b)

Fig. 6.- a) Estimated speed for different values of the speed using $\bar{B}_{x d q s}^{s}$ and b) $\bar{B}_{y d q s}^{s}$. Sensor position: $\mathrm{x}=0 \mathrm{~mm}, \mathrm{y}=0 \mathrm{~mm}, \mathrm{z}=5 \mathrm{~mm}$. $i_{d q}=0 \mathrm{pu}$.

The estimated position and the estimation error (position measured using a shaft encoder was used to obtain the estimation error) are shown in Fig. 5a and Fig. 5b, with no filtering stage implemented for these experimental results (see Fig. 2). It is observed that the position error is less than 3 electrical degrees when using $\bar{B}_{y d q s}^{r}$ for the estimation, and less than 4 electrical degrees when using $\bar{B}_{x d q s}^{r}$. As expected, the estimated position using $\bar{B}_{x d q s}^{r}$ has larger error due to its higher harmonic content.

Fig. 6a and Fig. 6b show the estimated speed using $\bar{B}_{x d q s}^{r}$ and $\bar{B}_{y d q s}^{r}$ respectively, for different values of the speed. No filtering stage has been implemented for these experimental results (see Fig. 2). It is observed from Fig. 6 that the noise in the speed estimation increases as the speed decreases. This is because the harmonics of the flux become spectrally closer to the fundamental component in this case, the PI controller of the PLL showing a higher gain to these components. It is concluded from this discussion that the PLL must be enhanced with harmonic rejection capabilities [40]-[41]. This could be achieved by adding a filtering stage to the PLL of the type shown by (3), or by pre-compensation of the harmonic components introduced by the sensors. This second option will be further discussed in the next section. Use of a filtering stage can be used in the mid-to-high speed region, but becomes ineffective at speeds below 5 to $7 \%$ of rated speed due to the overlap between the filter cut-off frequency and the fundamental frequency at $\omega_{r}$. This option cannot work therefore at standstill.

$B S F(s)=\frac{s-j n \omega_{r}}{s-j n \omega_{r}+\omega_{n}}$

Fig. 7a shows an example of the filtering stage that could be used in the mid-to-high speed region [40]. It consists of two band-stop filters, $B S F 1$ and $B S F 2$ defined by (3), where $n$ is the harmonic order, $\omega_{r}$ is the rotor electrical speed and $\omega_{n}$ determines the filter bandwidth. Fig. $7 \mathrm{~b}$ shows the frequency response of (3) for various bandwidths. BSF1 is used to reject the component at $-2 \omega_{r}$ in the rotor reference frame $\left(-\omega_{r}\right.$ in the stationary reference frame (see Fig. 3 and Fig. 4), being therefore frequency adaptive. This component is typically induced by unbalances in the sensors' gains, variation of their properties with temperature and assembling tolerances. BSF2 is used to reject the component at $-\omega_{r}$ in the rotor reference frame (DC component in the stator reference frame), which is typically induced by offsets in the sensors. For the experimental verification, $B S F 1$ and $B S F 2$ bandwidths have been set to $5 \mathrm{~Hz}$. All these issues will be further discussed in the next section.

a)
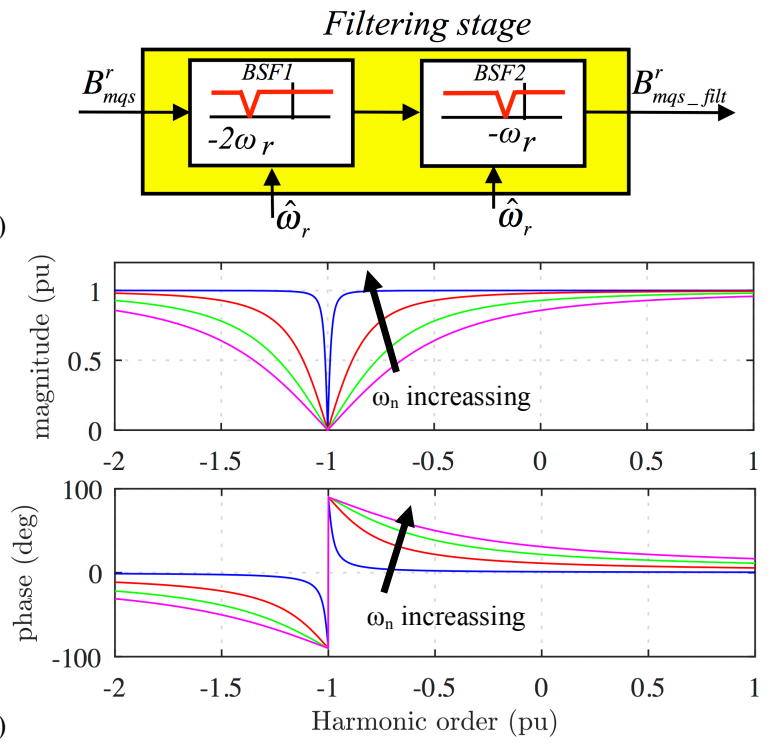

Fig. 7.- a) Filtering stage (see Fig. 2), and b) frequency response of a BSF, (3), when $n=1$ and $\omega_{\mathrm{n}}=5,2040$ and $60 \mathrm{rad} / \mathrm{s}$ respectively.
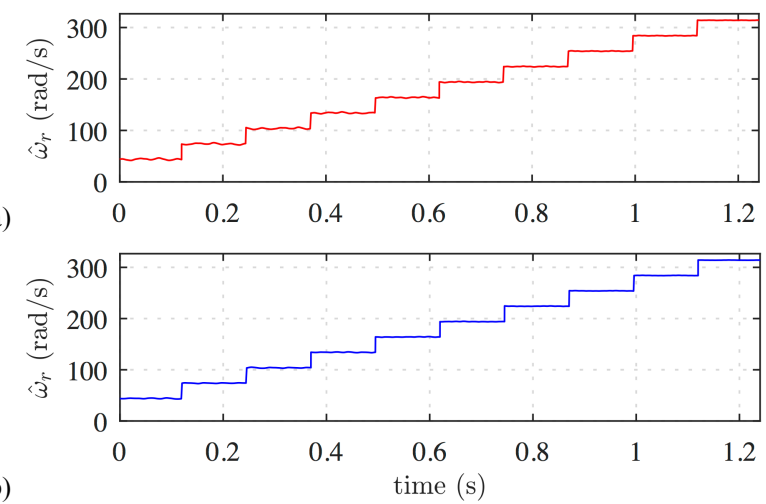

Fig. 8.- Same experimental results as in Fig. 6 when the filtering stage described in Fig. 7 is used. 


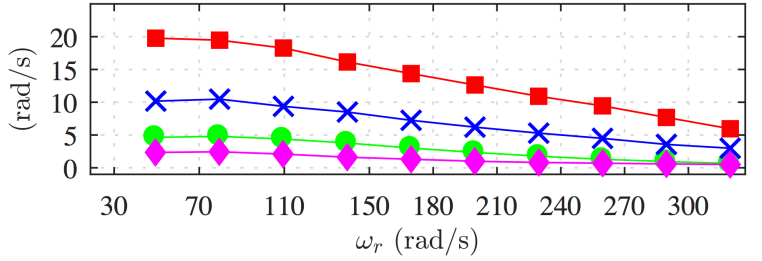

Fig. 9.- Peak-to-peak value of the estimated speed error for the experimental results shown in Fig. 6 and Fig. 8 using $\bar{B}_{x d q s}^{s}$, with $\left({ }^{\circ}\right)$ and without (ם) filtering stage; $\bar{B}_{v d a s}^{s}$ with (^) and without $(\times)$ filtering stage.

Fig. 8 shows the same experimental results as Fig. 6, when the filtering stage shown in Fig. 7 is implemented. Finally, the peak-to-peak values of the estimated speed error (speed measured using a shaft encoder was used to obtain the estimation error) for the experimental results shown in Fig. 6 and Fig. 8 with and without filtering are shown in Fig. 9. The improvements due to the filters are evident from this figure. It is also observed that the peak-to-peak value of the estimated speed error is significantly lower when using $\bar{B}_{y d q s}^{s}$ and that it increases as the speed decreases, especially when using $\bar{B}_{x d q s}^{s}$.

\section{Implementation issues}

There are a number of issues, which affect to the performance of the method and must therefore be considered. These include among others: 1) offsets in the sensors, 2) variations in the sensors' gains; 3) temperature dependence of the sensors, 4) assembling tolerances, 5) stator current effects. All these issues are analyzed following.

\section{III.A. Effect of offset in the sensors}

Offsets in the sensors measurements $\left(\Delta B_{\text {mas }}, \Delta B_{m b s}, \Delta B_{m c s}\right)$ can be modeled as shown in (4)-(5). It is observed from (5) that $\bar{B}_{\text {mdqs }}^{s}$ can be decomposed into two components: a fundamental component ( $\bar{B}_{d q s_{-} 1 \omega r}^{s}$ ), which rotates at the machine speed, and a DC component $\left(\Delta \bar{B}_{d q s_{3} \text { offset }}^{s}\right)$ whose magnitude and phase depend on the offsets. Fig. 10 shows the schematic representation of $\bar{B}_{m d q s_{-} 1 \omega r}^{s}, \Delta \bar{B}_{m d q s}^{s}$ and $\bar{B}_{m d q s}^{s}$, while Fig. 11 shows the magnetic flux density measured along $y$-axis by hall sensors aligned with phases $a, b$ and $c$, and the FFT of $\bar{B}_{y d q s}^{s}$ when $\Delta B_{y d q s}^{s}=0.1 B_{y d q s_{-} 1 \omega r}^{s}$.

$$
\begin{aligned}
\bar{B}_{m d q s}^{s} & =\frac{2}{3}\left(\begin{array}{l}
\left.\left(B_{\text {mas }}+\Delta B_{m a s}\right)+\left(B_{m b s}+\Delta B_{m b s}\right) e^{j 4 \pi / 3}+\right) \\
\left.\bar{B}_{m c s}^{s}+\Delta B_{m c s}\right) e^{j 2 \pi / 3}
\end{array}\right) \\
& =B_{m d q s_{-} 1 \omega r}^{s} e^{j \omega_{r} t}+\Delta B_{m d q s}^{s} e^{j \theta_{\Delta B}}= \\
& \bar{B}_{m d q s_{-} 1 \omega r}^{s}+\Delta \bar{B}_{m d q s_{-} O f f s e t}^{s} \\
\theta_{\text {error }} & =\arctan \left(\frac{\Delta B_{m d q s}^{s} \sin \left(\theta_{\Delta B}-\omega_{r} t\right)}{B_{m d q s_{-} 1 \omega r}^{s}+\Delta B_{m d q s}^{s} \cos \left(\theta_{\Delta B}-\omega_{r} t\right)}\right)
\end{aligned}
$$

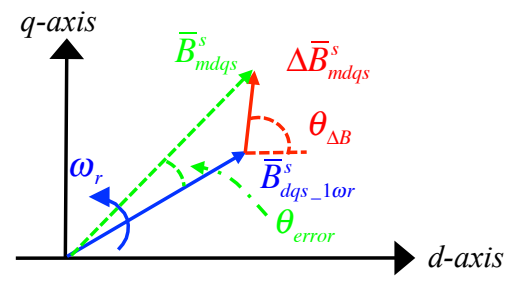

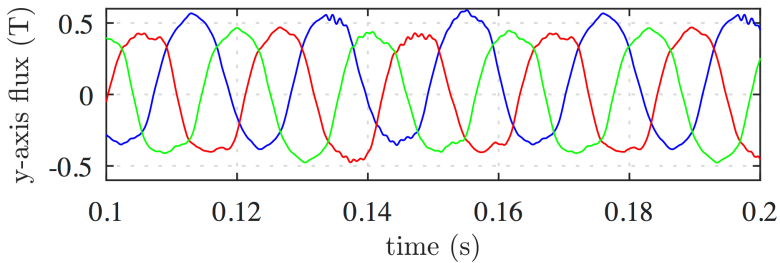

a)

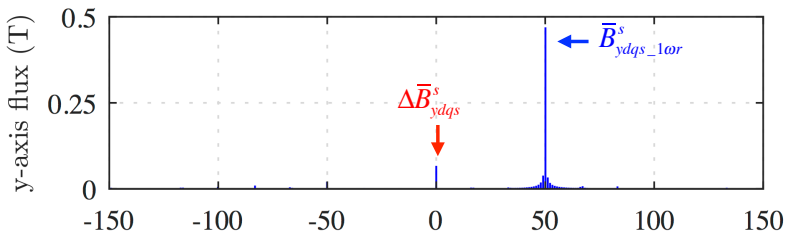

b)

$(\mathrm{Hz})$

Fig. 11.- a) Y-axis magnetic flux density measured by the sensors and b) FFT of the flux complex vector, $\bar{B}_{y d q s}^{s}$. Sensor position: $\mathrm{x}=0 \mathrm{~mm}, \mathrm{y}=0 \mathrm{~mm}$, $\mathrm{z}=5 \mathrm{~mm} . \omega_{r}=1 \mathrm{pu}, i_{d q}=0 \mathrm{pu}$ and $\Delta B_{y d q s}=0.1 B_{y d q s_{-} 1 \omega r}$.

It can be observed from Fig. 10 , that $\Delta \bar{B}_{m d q s}^{s}$ induces a position error (6). When the machine is rotating, $\Delta \bar{B}_{\text {mdqs }}^{s}$ can be easily eliminated from $\bar{B}_{m d q s}^{s}$ using e.g. a filter in the loop PLL [40], a MCCF-PLL [41] or any other PLL structure with harmonic rejection capability. However, decoupling of $\Delta \bar{B}_{m d q s}^{s}$ using linear filters is not possible when $\omega_{r}=0$ as in this case $\bar{B}_{m d q s_{-} 1 \omega r}^{s}$ and $\Delta \bar{B}_{m d q s}^{s}$ are DC quantities. Alternatively, a pre-commissioning process to calibrate the sensors can be used. The position error for initial position estimation can be expressed by (7), the maximum position error occurring whenever $\bar{B}_{m d q s_{-} 1 \omega r}^{s}$ and $\Delta \bar{B}_{m d q s}^{s}$ are $90 \mathrm{deg}$. phase shifted (see Fig. 10 and (8)). For example, the maximum position error is $\approx 5.71$ degrees for $\Delta B_{m d q s}^{s}=0.1 B_{m d q s_{-} 1 \omega r}^{s}$.

$\theta_{\text {error_init_max }}=\arctan \left(\frac{\Delta B_{m d q s}^{s}}{B_{m d q s_{-} 1 \omega r}^{s}}\right)$

$\theta_{\text {error_init }}=\arctan \left(\frac{\Delta B_{m d q s}^{s} \sin \theta_{\Delta B}}{B_{m d q s_{-} 1 \omega r}^{s}+\Delta B_{m d q s}^{s} \cos \theta_{\Delta B}}\right)$

\section{III.B. Effect of variation in the sensor gain}

Differences in sensors' gains ( $\Delta k_{a}, \Delta k_{b}$ and $\Delta k_{c}$ ) can be modeled as (9). Using the definition of the complex differential gain in (10), (11) is obtained. $\bar{B}_{\text {mdqs }}^{s}$ can be decomposed into three components (11): the desired component $\left(\bar{B}_{m d q s_{-} 1 \omega r}^{s}\right)$ which rotates at the machine speed, and a positive sequence component $\left(\Delta \bar{B}_{m d q s p c}^{s}\right)$ and a negative sequence component $\left(\Delta \bar{B}_{m d q s n c}^{s}\right)$ which rotate at $+\omega_{r}$ and $-\omega_{r}$ respectively, and whose magnitude and initial angle are $\Delta B_{m d q s}^{s}$ and $\varphi_{\Delta k}$ respectively. Fig. 12a shows the schematic representation of $\bar{B}_{m d q s_{-} 1 \omega r}^{s}, \Delta \bar{B}_{m d q s p c}^{s}, \Delta \bar{B}_{m d q s n c}^{s}$ and $\bar{B}_{m d q s}^{s}$, while Fig. 13 shows the magnetic flux density measured along $y$-axis and the FFT of $\bar{B}_{y d q s}^{s}$. Flux components $\bar{B}_{y d q s_{-} 1 \omega r}^{s}$, $\Delta \bar{B}_{y d q s p c}^{s}$ and $\Delta \bar{B}_{y d q s n c}^{s}$ are readily seen in the figure.

$\bar{B}_{m d q s}^{s}=\frac{2}{3}\left(\begin{array}{l}B_{m a s}\left(1+\Delta k_{a}\right)+B_{m b s}\left(1+\Delta k_{b}\right) e^{j 4 \pi / 3}+ \\ B_{m c s}\left(1+\Delta k_{c}\right) e^{j 2 \pi / 3}\end{array}\right)$

$\Delta \bar{k}=\left(\Delta k_{a}+\Delta k_{b} e^{j 4 \pi / 3}+\Delta k_{c} e^{j 2 \pi / 3}\right)=\Delta k e^{j \varphi_{\Delta k}}$

Fig. 10.- Schematic representation of $\bar{B}_{m d q s_{-} 1 \omega r}^{s}, \Delta \bar{B}_{m d q s}^{s}$ and $\bar{B}_{m d q s}^{s}$. 


$$
\begin{aligned}
\bar{B}_{m d q s}^{s} & =B_{m d q s_{-} 1 \omega r}^{s}\left(e^{j \omega_{r} t}+\frac{\Delta k}{2} e^{j\left(\omega_{r} t+\varphi_{\perp k}\right)}+\frac{\Delta k}{2} e^{j\left(-\omega_{r} t-\varphi_{\Delta k}\right)}\right) \\
& =B_{m d q s_{-} 1 \omega r}^{s} e^{j \omega_{r} t}+\Delta B_{m d q s p c}^{s} e^{j\left(\omega_{r} t+\varphi_{\perp k}\right)}+\Delta B_{m d q s n c}^{s} e^{j\left(-\omega_{r} t-\varphi_{\perp k}\right)} \\
& =\bar{B}_{m d q s_{-} 1 \omega r}^{s}+\Delta \bar{B}_{m d q s p c}^{s}+\Delta \bar{B}_{m d q s n c}^{s}
\end{aligned}
$$

$\Delta \bar{B}_{m d q s p c}^{s}$ and $\Delta \bar{B}_{m d q s n c}^{s}$ induce a position error given by (12). Decoupling of these components while the machine is rotating is significantly more challenging than the decoupling of components due to offsets, as $\Delta \bar{B}_{m d q s p c}^{s}$ and $\bar{B}_{m d q s_{-} 1 \omega r}^{s}$ are both at $\omega_{r}$. A modified PLL, e.g. a MCCF-PLL [41], capable of estimating the negative sequence component could be used; The estimated negative sequence component, $\Delta \bar{B}_{m d q s n c}^{s}$, could be used to decouple both $\Delta \bar{B}_{m d q s p c}^{s}$ and $\Delta \bar{B}_{m d q s n c}^{s}$ from $\bar{B}_{\text {mdqs }}^{s}$; note that $\Delta \bar{B}_{m \text { dqspc }}^{s}$ and $\Delta \bar{B}_{m d q s s c}^{s}$ have the same magnitude as well as the same phase shift with respect to $\bar{B}_{m d q s_{-} 1 \omega r}^{s}$ (see (11)).

$$
\begin{aligned}
& \theta_{\text {error }}= \\
& \arctan \left(\frac{\Delta B_{m d q s}^{s} \sin \varphi_{\Delta k}+\Delta B_{m d q s}^{s} \sin \left(-2 \omega_{r} t-\varphi_{\Delta k}\right)}{B_{m d q s_{-} 1 \omega r}^{s}+\Delta B_{m d q s}^{s} \cos \varphi_{\Delta k}+\Delta B_{m d q s}^{s} \cos \left(-2 \omega_{r} t-\varphi_{\Delta k}\right)}\right)
\end{aligned}
$$

As observed from (13) and Fig. 12b, $\Delta \bar{B}_{d q s p c}^{s}$ and $\Delta \bar{B}_{d q s n c}^{s}$ induce a position error when the machine does not rotate, the maximum initial position error being (14). For the hall-effect sensors used in this work [35], the error in the gains is $<0.01 \mathrm{pu}$, meaning that $\Delta B_{\text {mdqsnc }}^{s}=\Delta B_{\text {mdqspo }}^{s} \leq 0.005$, the maximum position error being therefore $\leq 0.57 \mathrm{deg}$.

$$
\begin{aligned}
& \theta_{\text {error_init }}= \\
& \arctan \left(\frac{\Delta B_{m d q s}^{s} \sin \varphi_{\Delta k}+\Delta B_{m d q s}^{s} \sin \left(-2 \theta_{r_{-} \text {init }}-\varphi_{\Delta k}\right)}{B_{m d q s_{-} \_r}^{s}+\Delta B_{m d q s}^{s} \cos \varphi_{\Delta k}+\Delta B_{m d q s}^{s} \cos \left(-2 \theta_{r_{-} i n i t}-\varphi_{\Delta k}\right)}\right) \\
& \theta_{\text {error_init_max }}=\arctan \left(\frac{\Delta B_{m d q s}^{s}}{B_{m d q s_{-} 1 \omega r}^{s}}\right)
\end{aligned}
$$

\section{III.C. Effect of sensor temperature}

Hall-effect sensor output voltage varies with its temperature [34]-[36], it can also be influenced by the circuit used to feed the sensor [34]-[36]. The output voltage vs. temperature for the hall-effect sensors used in this work is shown in Fig. 14a [35], the sensor is seen to be highly insensitive to temperature variations. However, this strongly depends on the sensor being used. Hall-effect sensors used in [34] were also evaluated (see Fig. 14b). It is observed from Fig. $14 \mathrm{~b}$ that the sensor is more sensitive to temperature when fed from a constant voltage source when compared to when the sensor is fed from a constant current source. It is finally noted that temperature compensated hall-effect sensors are commercially available [37]-[39]. a)

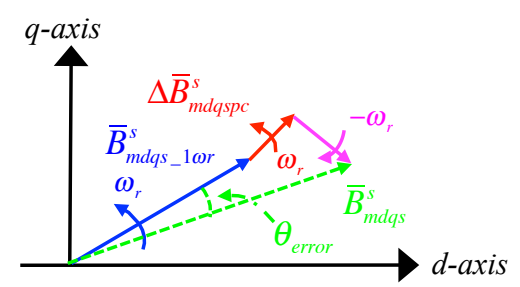

b)

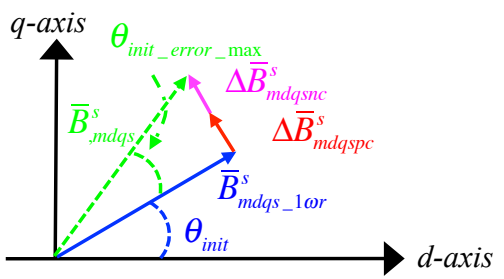

Fig. 12.- a) Schematic representation of $\bar{B}_{m d q s_{-} 1 \omega r}^{s}, \Delta \bar{B}_{m d q s p c}^{s}, \Delta \bar{B}_{m d q s n c}^{s}$ and $\bar{B}_{m d q s}^{s}$, and b) maximum position error at standstill due to variations in sensors' gains.
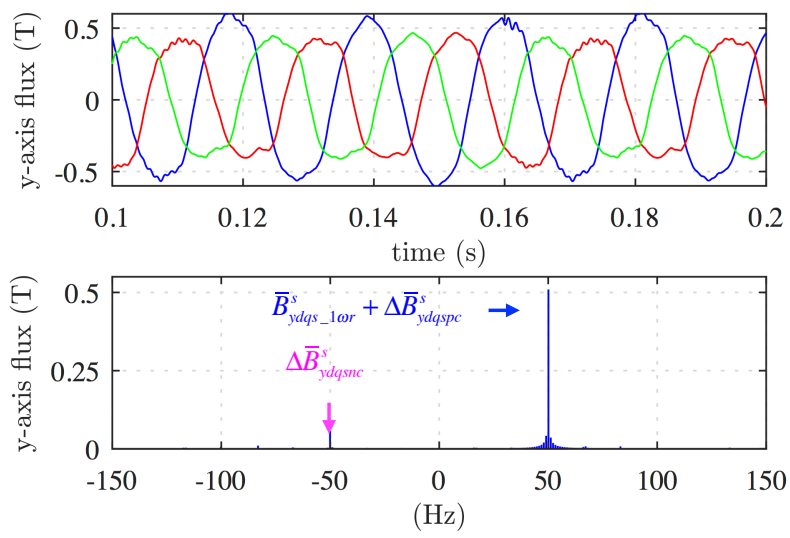

Fig. 13.- a) Y-axis magnetic flux density measured by sensors aligned with phases a, b and c, and b) FFT of the y-axis flux complex vector, $\bar{B}_{y d q s}^{s}(11)$, b). Sensor position: $\mathrm{x}=0 \mathrm{~mm}, \mathrm{y}=0 \mathrm{~mm}, \mathrm{z}=5 \mathrm{~mm} . \omega_{r}=1 \mathrm{pu}, i_{d q}=0 \mathrm{pu}$ and $\Delta k=0.1$.

It can be concluded from [34]-[36] and Fig. 14 that a variation of the hall-effect sensor temperature could be modeled as a change of the sensor gain. If there is no temperature difference among all three hall-effect sensors, changes in temperature will induce a variation of $\bar{B}_{m d q s}^{s}$, but this will not induce additional harmonic components and therefore no position error (12)-(13). This is the expected behavior in practice. On the contrary, temperature unbalances among sensors will induce additional harmonic components in $\bar{B}_{m d q s}^{s}$, and consequently errors in the estimated position of the type shown in (12)-(13). 


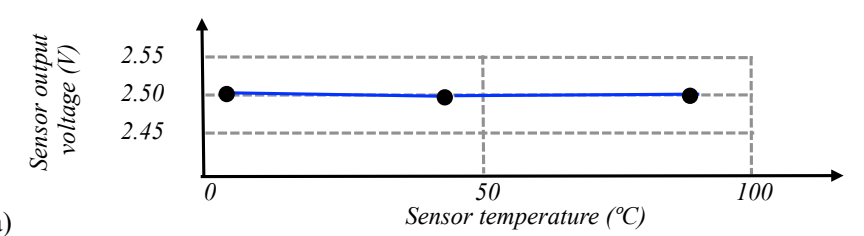

a)

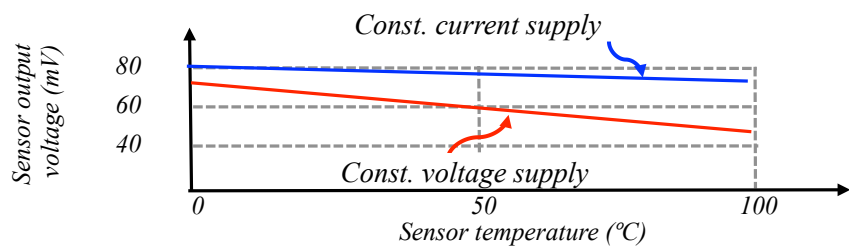

b)

Fig. 14.- Hall-effect sensor sensitivity for: a) sensor in [35] fed with constant voltage of $5 \mathrm{~V}$ and b) sensor in [34] fed with a constant voltage of $6 \mathrm{~V}$ and constant current of $10 \mathrm{~mA}$ respectively; flux density being applied to the sensor was $50 \mathrm{mT}[34]$.

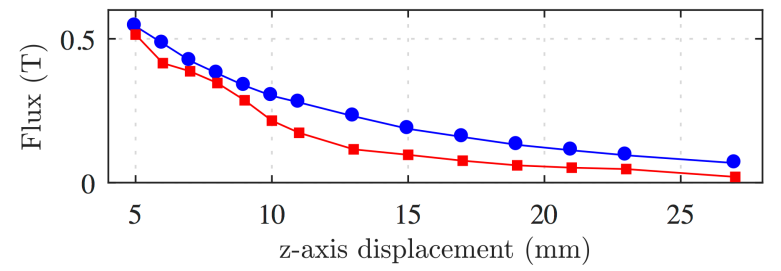

Fig. 15.- Peak value of $x(\bullet)$ and $y$-axis $(\bullet)$ magnetic flux measurements by hall effect sensor " $a$ ", see Fig. 1, as a function of the axial displacement. Sensor position: $\mathrm{x}=0 \mathrm{~mm}, \mathrm{y}=0 \mathrm{~mm} . \omega_{r}=1 \mathrm{pu}, I_{d q}=0 \mathrm{pu}$.

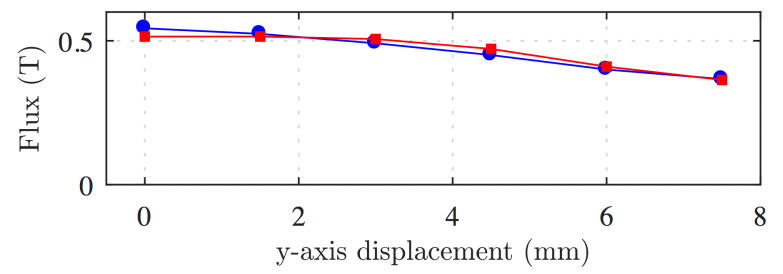

Fig. 16.- Peak value of $x(\bullet)$ and $y$-axis $(\bullet)$ magnetic flux measurements by hall effect sensor " $a$ ", see Fig. 1, as a function of the radial displacement (i.e. $\mathrm{y}$-axis). Sensor position: $\mathrm{x}=0 \mathrm{~mm}, \mathrm{z}=5 \mathrm{~mm}$. $\omega_{r}=1 \mathrm{pu}, I_{d q}=0 \mathrm{pu}$.

\section{III.D. Effect of assembling tolerances}

Assembling tolerances will result in unexpected displacements in axial, radial and angular directions. These three types of displacements are analyzed following.

\section{a) Axial displacement}

An axial displacement induces a variation of the magnetic flux density measured by the sensor both in $x$ and $y$-axis directions, where the measured magnetic flux densities decrease as the sensor is moved further away from the magnet, as shown in Fig. 15. This variation can be modeled as a variation of the sensor's gain, which has been previously analyzed in section IV-a.

\section{b) Radial displacement}

A radial displacement induces a variation of the magnetic flux density measured by the sensor in both $x$ and $y$-axis directions (see Fig. 16); it can be therefore modeled as a variation in the sensor gain, which has been previously analyzed in section IV-a. It can be observed from Fig. 15 and Fig. 16 that radial displacements produce a much lower variation of the magnetic flux density compared to axial displacements.

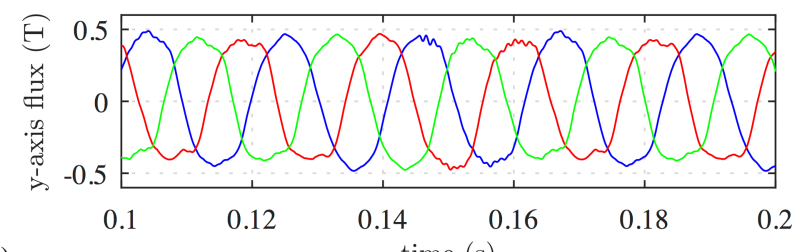

a)

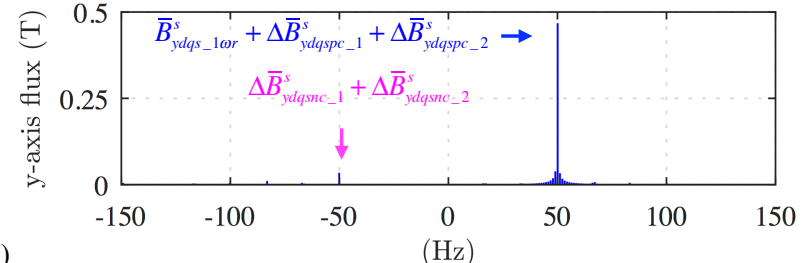

Fig. 17.- a) y-axis magnetic flux density measured by sensors aligned with phases a, b and c, and b) FFT of the y-axis flux complex vector, $\bar{B}_{y d q s}^{s}(16)$, b). Sensor position: $\mathrm{x}=0 \mathrm{~mm}, \mathrm{y}=0 \mathrm{~mm}, \mathrm{z}=5 \mathrm{~mm} . \quad \omega_{r}=1 \mathrm{pu}, \quad i_{d q}=0 \mathrm{pu}$, $\varphi_{\text {disp_a }}=10 \mathrm{deg}$., $\varphi_{\text {disp } \__{-}}=0 \mathrm{deg}$. and $\varphi_{\text {disp_c }}=0 \mathrm{deg}$. .

\section{c) Angular displacement}

Angular displacements of the sensors, $\varphi_{\text {disp_a }}, \varphi_{\text {disp_ } b}$ and $\varphi_{\text {disp_c }}$, can be modeled as (15). Using some basic trigonometric transformations, (15) can be expressed as (16). Comparing (11) and (16), it is observed that two additional harmonic components, $\Delta \bar{B}_{\text {mdqsp__2 }}^{s}$ and $\Delta \bar{B}_{\text {mdqsnc_2 }}^{s}$, shifted $\pi / 2$ from $\Delta \bar{B}_{m d q s p c_{-} 1}^{s}$ and $\Delta \bar{B}_{m d q s n c_{-} 1}^{s}\left(\Delta \bar{B}_{m d q s p c}^{s}\right.$ and $\Delta \bar{B}_{m d q s n c}^{s}$ in (11)), are induced, see Fig. 17. For example, the maximum induced position error is $\approx 6.6 \mathrm{deg}$. for a phase displacement of 10 mechanical degrees.

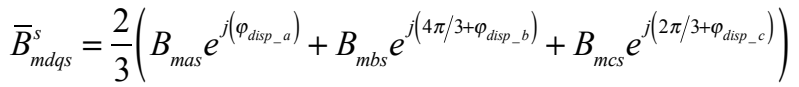

$$
\begin{aligned}
& \bar{B}_{m d q s}^{s}=B_{m d q s_{-} 1 \omega r}^{s} e^{j \omega_{r} t}+ \\
& +\left(\Delta B_{m d q s_{-} 1}^{s} e^{j\left(\omega_{r} t+\varphi_{\Delta k 1}\right)}+\Delta B_{m d q s_{-} 1}^{s} e^{j\left(-\omega_{r} t-\varphi_{\Delta k 1}\right)}\right)+ \\
& +\left(\Delta B_{m d q s_{-} 2}^{s} e^{j\left(\omega_{r} t+\varphi_{\Delta k 2}+\pi / 2\right)}+\Delta B_{m d q s_{-} 2}^{s} e^{j\left(-\omega_{r} t-\varphi_{\Delta k 2}+\pi / 2\right)}\right)= \\
& =\bar{B}_{m d q s_{-} 1 \omega r}^{s}+\Delta \bar{B}_{m d q s p c_{-} 1}^{s}+\Delta \bar{B}_{m d q s n c_{-} 1}^{s}+\Delta \bar{B}_{m d q s p c_{-} 2}^{s}+\Delta \bar{B}_{m d q s n c_{-} 2}^{s}
\end{aligned}
$$

It can be concluded from the previous discussion that the effects of sensor's assembling tolerances can influence the performance and require therefore some type of compensation.

\section{III.E. Effects of stator current}

The flux measured by the hall-effect sensors will be the result of the combined effect of PMs flux and the flux induced by the stator $d$ and $q$-axis currents.

a) Stator d-axis current 


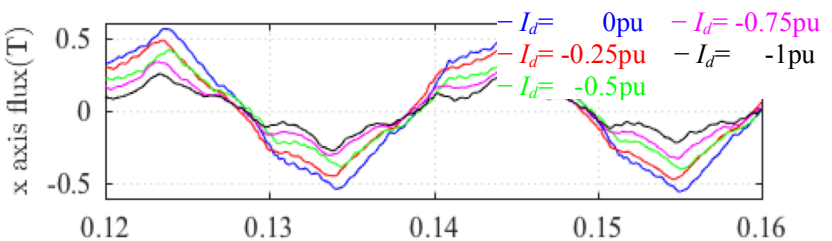

a)

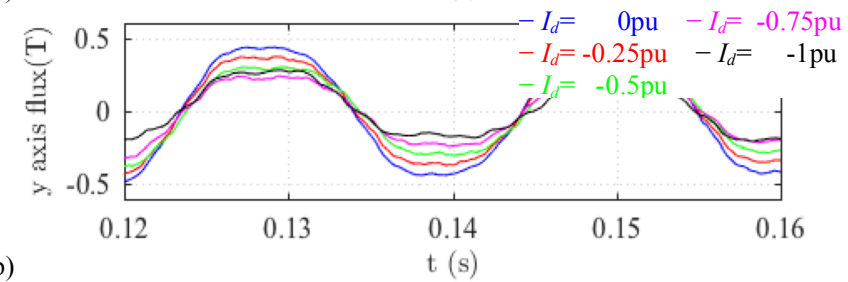

Fig. 18.- a) $x$-axis flux measurements by hall effect sensors " $a$ ", see Fig. 1; b) $y$-axis flux measurements by hall effect sensors " $a$ ", see Fig. 1. Sensor position: $\mathrm{x}=0 \mathrm{~mm}, \mathrm{y}=0 \mathrm{~mm}, \mathrm{z}=5 \mathrm{~mm}$. $\omega_{r}=1 \mathrm{pu}, I_{q}=0 \mathrm{pu}$.

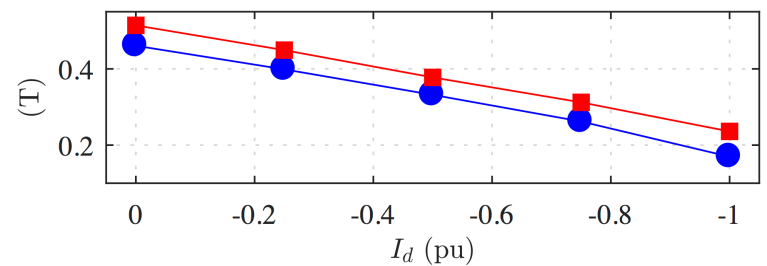

Fig. 19.- $\left|\bar{B}_{m d q s}^{r}\right|\left(-\left|\bar{B}_{x d q s}^{r}\right|\right.$ and $\left.\bullet\left|\bar{B}_{y d q s}^{r}\right|\right)$ vs. $I_{d}$. Sensor position: $\mathrm{x}=0 \mathrm{~mm}$, $\mathrm{y}=0 \mathrm{~mm}, \mathrm{z}=5 \mathrm{~mm}$. $\omega_{r}=1 \mathrm{pu}, I_{q}=0 \mathrm{pu}$.

$d$-axis current in PMSMs is typically defined to be aligned with the PM flux [24]-[33]. Negative $d$-axis current is used in IPMSMs below rated speed to implement MTPA control strategies while it is used in both SPMSMs and IPMSMs to allow operation above rated speed. Flux due to negative $d$ axis current partially counteracts the PM flux (fluxweakening current), resulting in an overall reduction of the rotor $d$-axis flux. This is expected to cause a reduction in the magnetic flux density measured by the hall-effect sensors. Fig. 18 shows the magnetic flux density measured by Hall-effect sensor " $a$ " (see Fig. 1), when negative $d$-axis current is applied and the $q$-axis current is equal to zero. It is observed that the peak value of the magnetic flux density decreases as negative $I_{d}$ increases. Fig. 19 shows the variation of $\left|\bar{B}_{x d q s}^{r}\right|$ and $\left|\bar{B}_{y d q s}^{r}\right|$ as the $d$-axis current changes from 0 to $-1 \mathrm{pu}$. It is observed that both $\left|\bar{B}_{x d q s}^{r}\right|$ and $\left|\bar{B}_{y d q s}^{r}\right|$ decrease as the current becomes more negative, the reduction being slightly higher in $y$-axis direction. It is also observed from Fig. 18 that the $d$-axis current does not cause phase shift in the measured magnetic flux density either in $x$ or $y$-axis directions, implying that it does not influence the estimated rotor position. This result was expected since the flux due to the $d$-axis current is in phase with the PM flux. However, generally speaking, the induced flux due to $I_{d}$ current injection could interfere with the $q$-axis flux due to the cross-coupling that typically exists between $d$ and $q$ axes, which will result in an error in the estimated position. For this particular machine, and based on the results shown in Fig. 18, cross-coupling between $d$ and $q$-axes can be safely neglected. Finally, it has been observed that $I_{d}$ current injection does not induce significant variation in $x$ or $y$-axis magnetic flux densities harmonic content, meaning that changes in the harmonic compensation method are not required.

It is concluded from the previous discussion that effects of $I_{d}$ current injection can be safely neglected.

\section{b) Stator q-axis current}

Flux induced by $I_{q}$ current is shifted from the PM flux by 90 electrical degrees. Therefore, $q$-axis current is expected to induce a variation both in the magnitude and phase of the measured magnetic flux density complex vector $\bar{B}_{m d q s}^{r}$. Furthermore, cross-coupling between $d$ and $q$-axes produce an interference with the PM flux, eventually resulting in additional magnitude and phase variations of $\bar{B}_{\text {mdqs }}^{r}$. However, as demonstrated in Fig. 18, this effect can be neglected for this particular machine. Fig. 20 shows the magnetic flux density measured by Hall-effect sensor " $a$ " (see Fig. 1), when $I_{q}$ current is being injected and $I_{d}=0 \mathrm{pu}$. It is observed that $I_{q}$ current induces a phase displacement of the magnetic flux density waveforms (the phase shift increasing as $I_{q}$ current does), which will result in an error in the estimated position. A slight increase of the harmonic content in both $x$ and $y$-axis directions is also observed. Fig. 21 shows the phase shift between $\bar{B}_{m d q s}^{r}\left(I_{q}=0\right)$ and $\bar{B}_{m d q s}^{r}\left(I_{q} \neq 0\right)$. It is observed that the induced phase shift in $x$-axis direction is slightly lower than in the $y$-axis direction. It can be concluded from the previous discussion that the

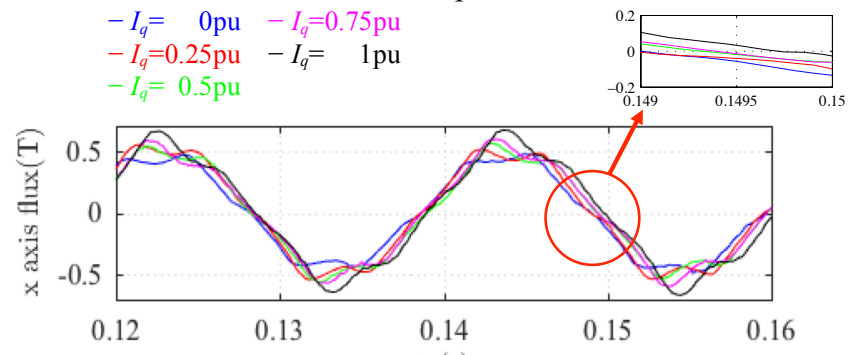

a)

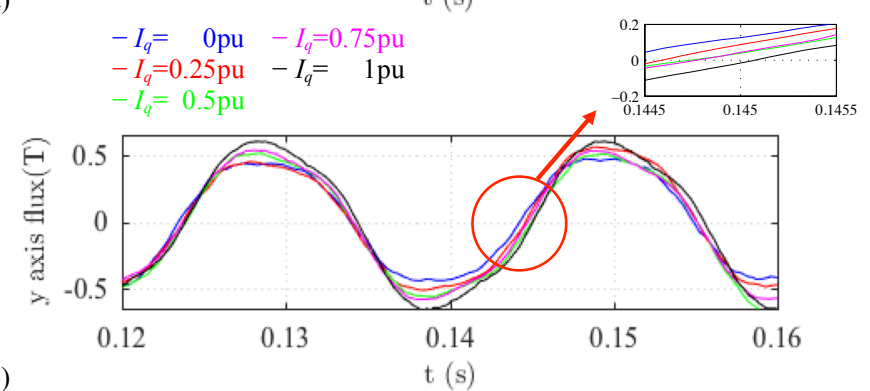

Fig. 20.-a) $x$-axis flux measurements by hall effect sensors " $a$ ", see Fig. 1; b) $y$-axis flux measurements by hall effect sensors " $a$ ", see Fig. 1. Sensor position: $\mathrm{x}=0 \mathrm{~mm}, \mathrm{y}=0 \mathrm{~mm}, \mathrm{z}=5 \mathrm{~mm}$. $\omega_{r}=1 \mathrm{pu}, I_{d}=0 \mathrm{pu}$.

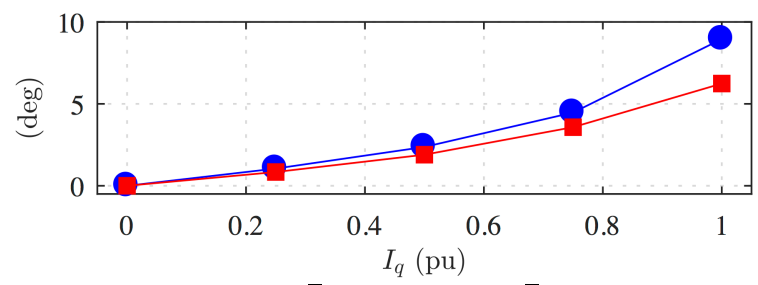

Fig. 21.- Phase shift between $\bar{B}_{m d q s}^{r}\left(I_{q}=0\right)$ and $\bar{B}_{m d q s}^{r}\left(I_{q} \neq 0\right) \quad(-x$-axis and - $y$-axis) vs. $I_{q}$. Sensor position: $\mathrm{x}=0 \mathrm{~mm}, \mathrm{y}=0 \mathrm{~mm}, \mathrm{z}=5 \mathrm{~mm} . \omega_{r}=1 \mathrm{pu}, I_{d}=0 \mathrm{pu}$. 

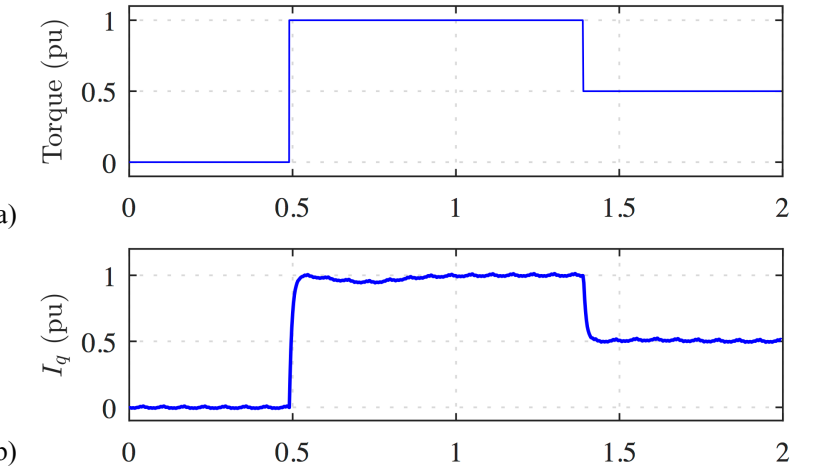

b)

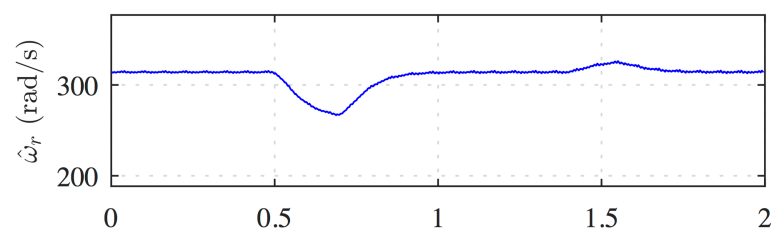

c)

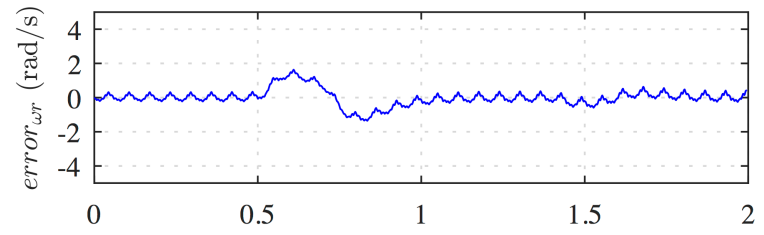

d)

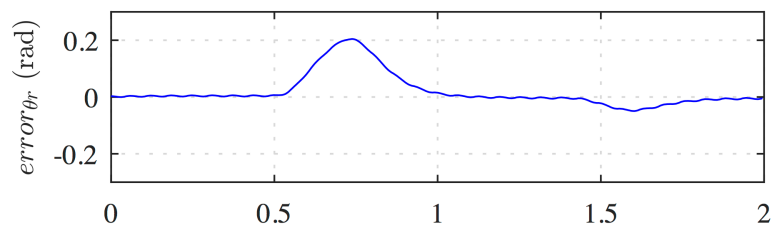

e)

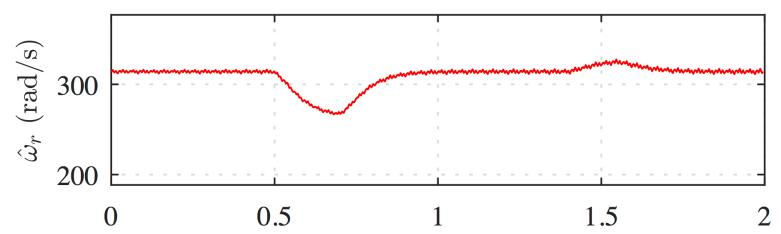

f)

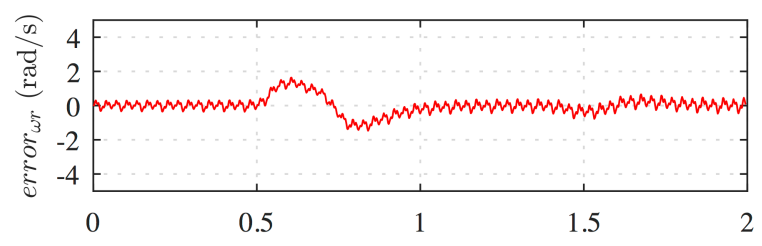

g)

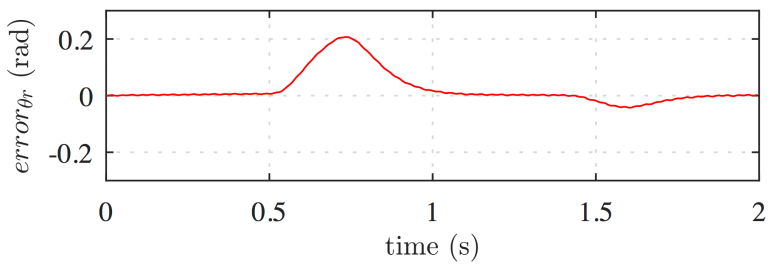

Fig. 22.-. Experimental results showing the transient response. a) Applied load torque and b) $I_{q}$. c), d) and e) estimated speed, speed error and position error using $\bar{B}_{y d q s}^{s}$. f), g) and h) Same results using $\bar{B}_{x d q s}^{s}$. Speed/position measured using a shaft encoder were used to obtain the estimation errors.

effects of $I_{q}$ current injection cannot be neglected. However, the phase shift due to $I_{q}$ current injection can be easily estimated and stored in a pre-commissioning process. The stored data can be accessed later during normal operation to implement on-line decoupling. It is finally observed that the harmonic content increase is more relevant in $x$-axis direction than in $y$-axis direction. As previously stated in Section 0, when the machine is rotating, i.e. $\omega_{r} \neq 0$, induced harmonics can be easily decoupled using a PLL with harmonic rejection capability [40]-[41]. However, decoupling of these harmonics using these methods is not possible when $\omega_{r}=0$, as all harmonics overlap as DC quantities in this case. A pre-commissioning process could be used in this case as well.

Finally, Fig. 22 shows the transient response when step-like torque variations are commanded to the drive; the machine was rotated by a prime mover and fed from a current regulated power converter using the estimated position for coordinate rotations. Fig. $22 \mathrm{~b}$ shows the injected $I_{q}$ current. Fig. 22c, $d$ and e show the estimated speed, speed error and position error when using $\bar{B}_{y d q s}^{r}$ for the estimation; Fig. 22f, g and $\mathrm{h}$ show the results when $\bar{B}_{x d q s}^{r}$ is used. It is observed that the estimated speed is noisier when using $\bar{B}_{x d q s}^{r}$ compared to $\bar{B}_{y d q s}^{r}$, which was expected from the results shown in Fig. 20. Speed and position errors are seen to be negligible in steady state, slightly increasing during the transients.

\section{Conclusions}

Control of PMSM drives using low cost analog hall-effect sensors for position/speed feedback was investigated in this paper. The principles of the magnetic flux density complex vector have been discussed first. Experimental results show that hall-effect sensors allow estimation of the rotor angle with adequate resolution, avoiding the use of speed/position sensors as encoders or resolvers. Implementation issues have also been thoroughly analyzed. It is concluded from this analysis that the effects of offsets, gains unbalances and temperature could be neglected in most of the applications, however effects of sensor's assembling tolerances and $I_{q}$ current injection need to be carefully evaluated and may need to be compensated.

Compared to other sensors as encoders or resolvers used for position feedback, the method proposed in this paper is advantageous in terms of cost, robustness and size as: 1) uses significantly cheaper sensors; 2) avoids moving parts and 3) does not require mechanical coupling to the machine shaft.

\section{Appendix I: Tuning of SRF-PLL}

The linearized model of an SRF-PLL for small errors in the estimated position is shown in Fig. 23 [45]-[48], the resulting closed loop transfer function being (17), where $K_{p}$ and $K_{i}$ are the proportional an integral gains of the PI controller.

$G_{c l}(s)=\frac{\hat{\theta}_{r}(s)}{\theta_{r}(s)}=\frac{K_{p} s+K_{i}}{s^{2}+K_{p} s+K_{i}}$

Gains $K_{i}$ and $K_{p}$ are normally selected to provide the desired bandwidth and to prevent oscillatory behavior (overshoots). Fig. 24a and b show the settling time and overshoot of (17) as a function of the controller gains. The 
response is mainly determined by the proportional gain $K_{p}$. It is interesting to note that for small values of $K_{p}$, the PLL can show inadmissible overshoots; large values of $K_{p}$ would then be preferred in principle, as this improves both overshoot and settling time (increases the bandwidth). However, excessive large values of $K_{p}$ can have adverse effects, as the PLL will become sensitive to harmonics and noise in general in the input signal. The gains of the PLL must therefore be chosen to traded-off its dynamic response and noise rejection capabilities, $K_{p}=80$ and $K_{i}=110$ have been used for the results presented in this paper.

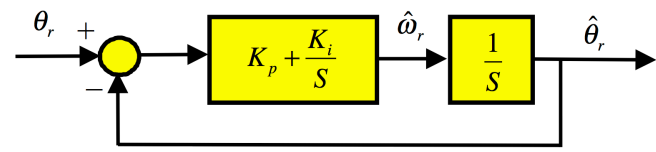

Fig. 23.-. Linearized model of the SRF-PLL.

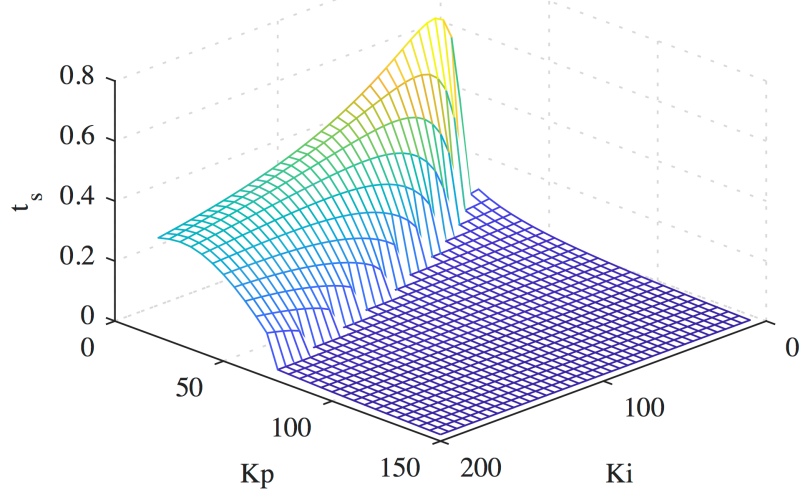

a)

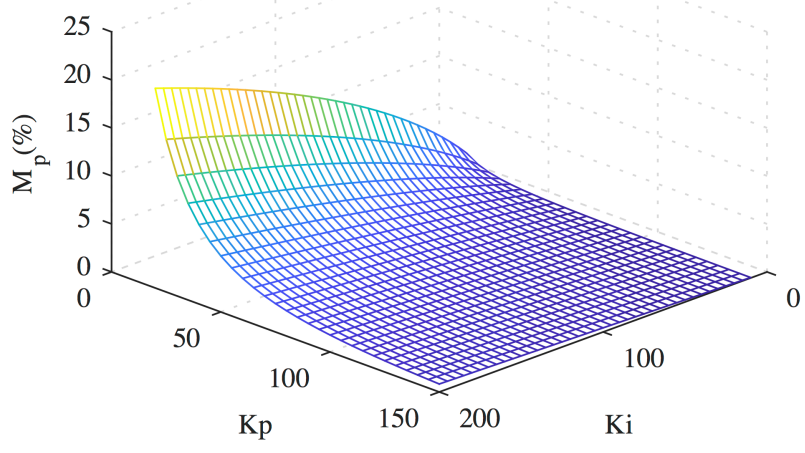

Fig. 24.-. a) Settling time and b) overshoot of $G_{c l}(s)$ vs. $K_{i}$ and $K_{p}$.

\section{References}

[1] D. W. Novotny and T. A. Lipo, "Vector Control and Dynamics of AC Drives," Oxford Science Publications, 1996.

[2] R. Nalepa and T. Orlowska-Kowalska, "Optimum trajectory control of the current vector of a non-salient pole PMSM in the field-weakening region," IEEE Trans. Ind. Electron., vol. 59, no. 7, pp. 2867-2876, Jul. 2012.

[3] G. Pellegrino, E. Armando, and Paolo Guglielmi, "Direct-Flux Vector Control of IPM Motor Drives in the Maximum Torque Per Voltage Speed Range," IEEE Trans. Ind. Appl., vol. 59, no. 10, pp. 3780-3788, Oct. 2012.
[4] B. Cheng and T. Tesch, "Torque feedforward control technique for permanent-magnet synchronous motors," IEEE Trans. Ind. Electron., vol. 57, no. 3, pp. 969-974, Mar. 2010.

[5] G. Pellegrino, E. Armando, and P. Guglielmi, "Direct flux field-oriented control of IPM drives with variable dc link in the field-weakening region," IEEE Trans. Ind. Appl., vol. 45, no. 5, pp. 1619-1627, Sep./Oct. 2009.

[6] Maxonmotorusa.com, "maxon sensor - Key information," Mar. 23, 2017. [Online]. Available: http://www.maxonmotorusa.com/

[7] Siemens.com, "Three-phase synchronous motors based on permanent magnet technology," Catalog D 86.2 • 2007, Mar. 23, 2017. [Online]. Available: http://w3.siemens.com/mcms/mc-solutions/en/motors/

[8] Technosoft.com, "Drive solutions for motion control applications," Product Overview, Mar. 23, 2017. [Online]. Available: http://www.technosoftmotion.com/en/products

[9] abbmotion.com, "Inteligent Servo Drives and Inteligent Motors," MotiFelex e180 motion control, Mar. 23, 2017. [Online]. Available: http://www.abbmotion.com/products/servodrives/overview.asp

[10] A. Yoo, S.-K. Sul, D.-C. Lee, and C.-S. Jun, "Novel speed and rotor position estimation strategy using a dual observer for low-resolution position sensors," IEEE Trans. Power Electron., vol. 24, no. 12, pp. 2897-2906, Dec. 2009

[11] M.C. Harke, G. De Donato, F. Giulii Capponi, T.R. Tesch, and R.D. Lorenz, "Implementation issues and performance evaluation of sinusoidal, surface-mounted PM machine drives with Hall-effect position sensors and a vector-tracking observer," IEEE Trans. Ind. Appl., vol. 44, no. 1, pp. 161 - 173, Jan./Feb. 2008.

[12] A. Lidozzi, L. Solero, F. Crescimbini, and A. Di Napoli, "SVM PMSM drive with low resolution Hall-effect sensors," IEEE Trans. Power Electron., vol. 22, no. 1, pp. 282 - 290, Jan. 2007.

[13] S. Kim, C. Choi, K. Lee, and W. Lee, "An improved rotor position estimation with vector-tracking observer in PMSM drives with lowresolution hall-effect sensors," IEEE Trans. Ind. Electron., vol. 58, no. 9, pp. 4078 - 4086, Sep. 2011.

[14] Y. Liu, J. Zhao, M. Xia, and H. Luo, "Model reference adaptive controlbased speed control of brushless DC motors with low-resolution halleffect sensors," IEEE Trans. Power Electron., vol. 29, no. 3, pp. 1514 1522, Mar. 2014

[15] B. B. Philip, D. P. Steven, J. D. Bradley, and C. K. Andreas, "Compensation for asymmetries and misalignment in a Hall-effect position observer used in PMSM torque-ripple control," IEEE Trans. Ind. Appl., vol. 43, no. 2, pp. 560-570, Mar./Apr. 2007.

[16] S. Morimoto, M. Sanada, and Y. Takeda, "Sinusoidal current drive system of permanent magnet synchronous motor with low resolution position sensor," in Proc. IEEE IAS Annual Meeting, San Diego, CA, Oct. 1996, pp. 9-13.

[17] S. Morimoto, M. Sanada, and Y. Takeda, "High performance currentsensorless drive for PMSM and SynRM with only low resolution position sensor," IEEE Trans. Ind. Appl., vol. 39, no. 3, pp. 792-801, May/Jun. 2003.

[18] F. G. Capponi, G. D. Donato, L. D. Ferraro, O. Honorati, M. C. Harke, and R. D. Lorenz, "AC brushless drive with low-resolution hall-effect sensors for surface-mounted PM machines," IEEE Trans. Ind. Appl., vol. 42, no. 2, pp. 526-535, March/April 2006.

[19] Y. F. Shi, Z. Q. Zhu, and D. Howe, "EKF-based Hybrid Controller for Permanent Magnet Brushless Motors Combining Hall Sensors and a Flux-Observer-based Sensorless Technique," IEEE-IEMDC, pp. 14661472, May 2005.

[20] A. Simpkins, and E. Todorov, "Position Estimation and Control of Compact BLDC Motors Based on Analog Linear Hall Effect Sensors," American Control Conference, pp. 1948-1955, July 2010.

[21] X. Song, J. Fang, and B. Han, "High-Precision Rotor Position Detection for High-Speed Surface PMSM Drive Based on Linear Hall-Effect Sensors," IEEE Trans. Power Electron., vol. 31, no. 7, pp. 4720 -4731, July 2016.

[22] Y. Y. Lee, R.-H. Wu, and S.T. Xu, "Applications of Linear Hall-Effect Sensors on Angular Measurement," IEEE International Conference on Control Applications (CCA), pp. 479-482, Sept. 2011.

[23] L. Xiao, Y. Yunyue, Z. Zhuo, "Study of the Linear Hall-Effect Sensors Mounting Position for PMLSM," IEEE Conference on Industrial Electronics and Applications, pp. 1175 - 1178, May 2017.

[24] Z. Wang, K. Lu, and F. Blaabjerg, "A simple startup strategy based on current regulation for back-emf-based sensorless control of pmsm," 
IEEE Transactions on Power Electronics, vol. 27, no. 8, pp. 3817-3825, 2012.

[25] F. Genduso, R. Miceli, C. Rando, and G. R. Galluzzo, "Back EMF sensorless-control algorithm for high-dynamic performance PMSM," IEEE Trans. Ind. Electron., vol. 57, no. 6, pp. 2092-2100, Jun. 2010.

[26] B. N. Mobarakeh, F. Meibody-Tabar, and F.-M. Sargos, "Back-EMF estimation based sensorless control of PMSM: Robustness with respect to measurement errors and inverter irregularities," in Conf. Rec. 39th IEEE IAS'04, vol. 3, pp. 1858-1865, Sept. 2004.

[27] Z. Q. Zhu, Y. Li, D. Howe, C. M. Bingham, and D. Stone, "Influence of machine topology and cross-coupling magnetic saturation on rotor position estimation accuracy in extended back-EMF based sensorless PM brushless AC drives," IEEE IAS'07, pp. 2378-2385, Sept. 2007.

[28] Z. Chen, M. Tomita, S. Koki, and S. Okuma, "An extended electromotive force model for sensorless control of interior permanentmagnet synchronous motors," IEEE Trans. Ind. Electron., vol.50, no.2, pp. 288-295, Apr. 2003.

[29] S. Morimoto, K. Kawamoto, M. Sanada, and Y. Takeda, "Sensorless control strategy for salient-pole PMSM based on extended EMF in rotating reference frame," IEEE Trans. Ind. Appl., vol.38, no.4, pp. 1054-1061, July/Aug. 2002.

[30] J.-I. Ha, K. Ide, T. Sawa, and S.-K. Sul, "Sensorless rotor position estimation of an interior permanent-magnet motor from initial states," IEEE Trans. Ind. Appl., vol. 39, no. 3, pp. 761-767, May/Jun. 2003.

[31] P. Guglielmi, M. Pastorelli, and A. Vagati, "Cross-saturation effects in IPM motors and related impact on sensorless control," IEEE Trans. Ind. Appl., vol. 42, no. 6, pp. 1516-1522, Nov./Dec. 2006.

[32] D. Reigosa, P. García, D. Raca, F. Briz, and R. D. Lorenz, "Measurement and adaptive decoupling of cross-saturation effects and secondary salien- cies in sensorless-controlled IPM synchronous machines," IEEE Trans. Ind. Appl., vol. 44, no. 6, pp. 1758-1767, Nov./Dec. 2008.

[33] D. Reigosa, K. Akatsu, N. Limsuwan, Y. Shibukawa, and R. D. Lorenz, "Self-sensing comparison of fractional slot pitch winding vs. distributed winding for FW- and FI-IPMSMs based on carrier signal injection at very low speed," IEEE Trans. Ind. Appl., vol. 46, no. 6, pp. 2467-2474, Nov./Dec. 2010.

[34] D. Fernandez, D. Hyun, Y. Park, D. Reigosa, S. B. Lee, D. M. Lee and F. Briz, "Permanent Magnet Temperature Estimation in PM Synchronous Motors Using Low Cost Hall Effect Sensors," IEEE Trans. Ind. Appl., vol. 53, no. 5, pp. 4515-4525, Sept./Oct. 2017.

[35] http://www.allegromicro.com/en/Products/Magnetic-Linear-AndAngular-Position-Sensor-ICs/Linear-Position-Sensor-ICs/A1301-2.aspx

[36] http://www.hallsensors.de/CYSJ106C.pdf

[37] www.allegromicro.com/media/Files/A3517-Datashee

[38] http://www.hallsensors.de/CYL840X.pdf

[39] http://www.analog.com/media/en/technical-documentation/datasheets/AD22151.pdf

[40] C. Blanco, D. Reigosa, F. Briz and J. M. Guerrero, "Synchronization in Highly Distorted Three-phase Grids Using Selective Notch Filters," IEEE-ECCE'14, pp. 2641-2648, Sept. 2014.

[41] X. Guo, W. Wu, and Z. Chen, "Multiple-Complex Coefficient-FilterBased Phase-Locked Loop and Synchronization Technique for ThreePhase Grid Interfaced Converters in Distributed Utility Networks," IEEE Trans. on Ind. Electr., vol. 58, no. 4,pp. 1194-1204, Apr. 2011.

[42] David Reigosa, Daniel Fernandez, Cristina Gonzalez, Sang Bin Lee and Fernando Briz, "Permanent Magnet Synchronous Machine Drive Control Using Analog Hall-Effect Sensors," IEEE ECCE, pp. 39663971, Oct. 2017.

[43] Faulhaber.com, "Brushless Dc servomotors," Series 22...BX432 3268...BX4, Mar. 23, 2017. [Online]. Available: https://fmcc.faulhaber.com

[44] Omrom Industrial Automation, "G5 series servosystems", R88ACRKC005NR-E, Nov. 28, 2017. [Online]. Available: https://industrial.omron.eu/en/home

[45] Y. F. Wang and Y. W. Li, "Analysis and Digital Implementation of Cascaded Delayed-Signal-Cancellation PLL," IEEE Trans. Power Electron., vol. 26, no. 4, pp. 1067-1080, Apr. 2011.

[46] S. Golestan, J. M. Guerrero, "Conventional Synchronous Reference Frame Phase-Locked Loop Is An Adaptive Complex Filter" IEEE Trans. Ind. Electron., vol. 62, no. 3, pp. 1679-1682, July 2014.

[47] J. A. Suul, K. Ljøkelsøy and Tore Undeland, "Design, Tuning and Testing of a Flexible PLL for Grid Synchronization of Three-Phase Power Converters" IEEE EPE, pp. 1-10, July 2009.
[48] E. Bueno, F. Rodriguez, F. Espinosa, and S. Cobreces, "SPLL design to flux oriented of a VSC interface for wind power applications," in Proc. 31st Annu. Conf. IEEE Ind. Electron. Soc., 2005 (IECON 2005), Nov., pp. 2451-2456.

[49] ABB motors, "Low-voltage three-phase induction motor, M2QA225M2A", Nov. 28, 2017. [Online]. Available: https://www.abb.com/product 